\title{
Pricing and revenue management in fragmented and segmented supply chains
}

\author{
Vipul Jain ${ }^{1} \cdot$ Gajanan Panchal ${ }^{2}$
}

○ Springer Nature Limited 2019

\section{Introduction}

Complexity of pricing decision and revenue management (RM) increases with more fragmented supply chain with many third-party and fourth-party logistics partners. Different market segments add more complications to it ranging from aligning the pricing strategies keeping the objective of maximizing revenue to selecting supply chain partners in a fragmented environment. This is true, e.g. in an e-commerce market, where a cross-border supply chain involves suppliers, buyers, logistics service providers, and consumers. To manage pricing and revenue management decision in such environment requires diverse disciplines work together in coherence-operations research, management science, analytics, informatics, economics, data analytics, software development, marketing, economics, e-commerce, first and last mile logistics, and consumer behavior modeling. From a practitioner standpoint, RM encompasses a range of activities related to demand planning, price optimization, sales maximization, market segmentation, capacity and inventory allocation, and business process management. This special issue centers on this idea.

This special issue aims at putting market segmentation and fragmentation in the spotlight so that they can be better evaluated and analyzed in making the decision-making functionality stronger. The special issue welcomed academic papers (qualitative, quantitative, mixed methods), practitioner's papers, case studies, and other relevant contribution to produce a ground-breaking special issue dedicated to pricing and revenue management in fragmented and segmented supply chain environment. As mentioned, the contributions cover both aspects of theoretical and practical development regarding pricing and revenue management in fragmented

\footnotetext{
$\checkmark$ Vipul Jain

vipul.jain@vuw.ac.nz

Victoria University of Wellington, Wellington, New Zealand

2 National University of Singapore, Singapore, Singapore
}

and segmented supply chains. It is hard to draw precise conclusions and trends across the papers included in this special issue, but there is no doubt that the papers elaborate and refine many of the trends presented in the introduction of this note. As guest editors, we are very satisfied both with the quality of the papers presented in this issue, and with the relevance on the themes I hoped to put focus on via this special issue.

\section{Overview of papers selected}

It is worth mentioning that a well-designed supply chain configuration yields positive net value by creating benefits, reducing costs, and improving firm's profitability. Therefore, the first paper by Agarwal and Singh, proposes a multiobjective optimization model to derive an integrated net present value-based supply chain configuration for a manufacturing enterprise incorporating the effect of third-party logistics services providers in an uncertain demand scenario. Firstly, the paper presents the conceptual framework considering the third-party logistics services providers for a manufacturing enterprise and thereafter a multi-objective optimization model is proposed to find a compromise solution to NPV maximization and total cost minimization. The model also makes use of chance constraint methodology to handle demand uncertainties.

Rapid innovation in technology accelerates obsolescence and hence product life cycles are getting shorter and wastes build up faster. Remanufacturing of short life-cycle product is an important alternative to mitigate the waste. However, since there are impacts in implementing remanufacturing, such as cannibalization, segmentation, and lower willingness to pay, pricing has become a significant aspect to ensure successful remanufacturing business. The second paper by Gan et al. studies manufacturer's and retailer's pricing decision of short life-cycle product considering the green segment consumers and propose two scenarios, i.e., independently 
optimized profit scenario under Stackelberg game with manufacturer as the leader, and integrated scenario. The results show that the integrated scenario achieves higher profit compared to the independent one, and both players are better off under the integrated decision. The paper also find that the existence of green segment increases the profit of manufacturer and retailer to a certain level, before it erodes manufacturer's profit when the green level is too high. In addition, price sensitivity of new and remanufactured products, demand's speed of change, and remanufacturing cost influence the optimum prices as well as the optimum green level. For firms that are engaging in remanufacturing, managerial insights are also provided to assist managers in making pricing decisions when there exist green consumers.

Goods and Service Tax (GST), a destination based unified taxation system, was implemented on 1st July 2017 in India replacing 17 different indirect taxes with the vision to create a seamless common market. A country like India, with the population of over 1.3 billion and heterogeneous distribution of wealth having a federal structure, opted for six different tax slab and dual-GST structure. In some countries, GST has been successful but in others, it has failed. The third paper by Kumar, Barve and Yadav have attempted to investigate the barriers to the smooth implementation of GST. In this paper, we have identified 12 barriers to GST implementation. By using interpretive structural modeling (ISM), this study finds the driving and dependence nature of different barriers to develop a structural model. The results of analysis found that lack of skilled manpower, lack of clarity of GST provisions, political unwillingness, and lack of policy for proper division of tax are the major barriers in the implementation of GST. In addition to the above analysis, MICMAC analysis is utilized to cluster the barriers in four categories as per their relative driving and dependence powers.

The fourth paper by Adnan and Ozelkan analyzes the occurrence of Bullwhip effect under three game scenarios (e.g. a simultaneous, a wholesale-leading, and a retailleading) for three types of demand functions (e.g. a logconcave, an isoelastic, and a negative exponential). Cost pass-throughs and Bullwhip effect ratios are calculated analytically for an $\mathrm{N}$-stage supply chain, and then the price fluctuations in various supply chain game structures are illustrated through simulations. The results indicate that in the case of optimal markup pricing games, the occurrence of Bullwhip effect depends on the demand functions. This study also shows that, Bullwhip effect occurs in varying magnitudes for different types of games. Finally, a relation between price variations and corresponding markup profits are also discussed.

The key idea of the fifth paper by Das, Hui and Jain is the development of a probabilistic model for optimum inventory policy for a three-product, four-echelon, distributive system. The mathematical model is built based on integer policy of ordering in the deterministic part with continuous review inventory policy. It is found to be a non-linear multi-variable constrained optimization problem. For illustration purpose, demand is taken as stationery random one and lead-time is normally distributed. A MATLAB program is generated to derive the solution based on exhaustive search method. Optimum lot-sizes, safety stocks, reorder points and service levels for each product at each stage are found out in accordance with continuous review inventory system. Further inventory turnover ratio computed is found to be reasonable and bubble plot of chosen service levels of different products with annual sales, profit margin, variability in demand and lead-time is obtained with expected characteristics. The proposed model along with the applied method of solving can find the best possible order quantity and ROP of a stochastic inventory system efficiently.

Vipul Jain and Gajanan Panchal

(Guest Editors)

Acknowledgements We would like to thank all reviewers for providing in-depth comments and constructive criticisms, and the authors for contributing their high-quality manuscripts. Without your help, it would have been impossible to produce this special issue. Thanks, are also due to the Editor-in-Chief, Ian Yeoman for his advice and help in making this special issue possible.

Publisher's Note Springer Nature remains neutral with regard to jurisdictional claims in published maps and institutional affiliations. 